\title{
A Distribuição Normal-assimétrica como Modelo para Produtividade de Milho Aplicada ao Seguro Agrícola'
}

\author{
Caroline Oliveira Santos², João Domingos Scalon ${ }^{3}$ e \\ Vitor Augusto Ozaki ${ }^{4}$
}

Resumo: O seguro agrícola comercializado no Brasil usa como parâmetro a produtividade agrícola média municipal dos últimos quatro ou cinco anos para se determinar a produtividade garantida. Diversos pesquisadores consideram que usar um modelo probabilístico para a distribuição de produtividade agrícola é uma metodologia mais adequada para a precificação de risco. Este estudo propõe a distribuição normal-assimétrica como uma alternativa à distribuição normal para modelar a distribuição da produtividade agrícola no Brasil. Foram analisadas séries de produtividade de milho, no período de 1981 a 2007, em 30 municípios do Paraná. A distribuição normal-assimétrica apresentou melhores ajustes do que a distribuição normal para a grande maioria dos municípios e, consequentemente, acarretou melhores estimativas para o pagamento esperado do seguro agrícola.

Palavras-chaves: adequabilidade do ajuste, APH, pagamento esperado, séries temporais.

\begin{abstract}
The crop insurance sold in Brazil is estimated using the average of the municipal agricultural productivity of the last four or five years. Many researchers believe that using a probabilistic model for the distribution of agricultural productivity is a better methodology for this purpose. This study advocates using the skew-normal distribution as an alternative to the normal distribution to model the distribution of agricultural productivity in Brazil. We analyzed series of corn yield from 1981 to 2007, in 30 counties in Paraná State, Brazil.
\end{abstract}

1. Os autores agradecem aos dois revisores pelos comentários e sugestões que muito contribuíram para melhoria da qualidade do artigo. O presente trabalho foi financiado pelo projeto Procad/ CNPq. C.O.S. agradece à Capes e ao CNPq pela bolsa.

2. Professora do Centro Federal de Educação Tecnológica Celso Suckow da Fonseca (CEFET/RJ). E-mail: carolineoliviera@yahoo.com.br

3. Professor do Departamento de Ciências Exatas da Universidade Federal de Lavras (UFLA). E-mail: scalon@dex.ufla.br

4. Professor do Departamento de Economia, Administração e Sociologia da Escola Superior de Agricultura Luiz de Queiroz (ESALQ/USP) e Coordenador do GRUPO GESER - Gestão em Seguros e Riscos. E-mail: vitorozaki@gmail.com 
The skew-normal distribution showed better fits than the normal distribution for the vast majority of the counties, and, consequently, led to better estimates for the expected payment of crop insurance.

Key-words: goodness of fit, APH, expected payment, time series.

Classificação JEL: C22, Q19.

\section{Introdução}

A agricultura é uma das mais antigas atividades desempenhadas pelo ser humano. $\mathrm{O}$ desenvolvimento da agricultura fez com que ela se tornasse um grande negócio, fundamental para a existência humana. A agricultura é uma atividade desprovida de mecanismos de defesa contra ocorrências que fogem ao controle do produtor rural. As pragas e os eventos climáticos severos podem determinar uma queda na produtividade agrícola esperada. Conforme afirma Ozaki (2005), a quebra de safra pode lesar não só o produtor atingido, mas a dinâmica econômica de toda uma região ou, até mesmo, de um país, dependendo de quão bruscos e abrangentes sejam o evento climático ou a ocorrência de doenças e pragas.

A queda na produção agrícola cria sérios problemas para produtores que adquiriram empréstimo para custeio da safra, desencorajando-os a continuar na atividade. Por este motivo é importante que o governo atente para o equilíbrio do mercado de produtos agrícolas, assegurando preços mínimos para esses produtos. Os produtores desenvolveram alguns mecanismos de defesa e atenuação do risco, tais como a diversificação de culturas, a diversificação espacial e intersetorial e a utilização de seguros (OZAKI, 2005).

Ozaki (2006) afirma que o seguro é uma das formas mais eficazes de se transferir o risco dos produtores para outros agentes econômicos.
Tarifas precisas são essenciais para o bom desenvolvimento de um programa de seguros. Os prêmios de seguro se destinam a cobrir perdas ao longo do tempo. O que a seguradora procura fazer é estimar rendimentos de safras futuras, no intuito de prever as perdas esperadas e pagamentos.

Desde 2003, os produtores rurais brasileiros estão tendo maiores incentivos do governo federal, com a implementação do Programa de Subvenção ao Prêmio do Seguro Rural. Este programa visa reduzir o custo do seguro para o produtor rural, auxiliando-o com a subvenção de um percentual do valor do prêmio, incentivando e proporcionando, aos mesmos, maior estabilidade de renda. Estes incentivos geram condições favoráveis para o crescimento do setor de seguro agrícola, entretanto exige o desenvolvimento de meios para quantificar e precificar este tipo de seguro.

O seguro agrícola tem algumas peculiaridades que o diferencia de outros tipos de seguro, tais como falta de dados, deficiência de normatização e/ou agência normatizadora, difícil precificação, elevada exposição às catástrofes, alto custo de fiscalização e peritagem. Tais peculiaridades fazem com que o seguro agrícola tenha prêmios elevados. O que desestimula o desenvolvimento do mercado, tornando-o desinteressante para a iniciativa privada.

O seguro agrícola mais vendido no Brasil é o seguro de custeio, que assegura ao produtor uma 
produtividade mínima com um preço garantido. Este seguro tem como estimador a produtividade agrícola média municipal dos últimos quatro anos, fato que pode causar distorções no cálculo do prêmio do seguro.

Além disso, estudos também apontam que se as taxas de prêmio não estão bem definidas, a seguradora pode ter problemas de seleção adversa e risco moral (COBLE et al., 1997; JUST, CALVIN e QUIGGIN, 1999; OZAKI, 2005). Conforme descrito por Ozaki (2005), a seleção adversa ocorre quando a seguradora faz seguro para propriedades rurais com alta probabilidade de ocorrência de sinistros, não conseguindo distinguir entre os diferentes grupos de risco. A seleção adversa pode levar a seguradora à ruína. $\mathrm{O}$ risco moral se refere ao fato de que a decisão ótima do segurado pode mudar em função da aquisição do seguro. Ozaki (2005) afirma, ainda, que, de modo geral, o risco moral ocorre no seguro agrícola quando a firma seguradora é incapaz de observar se o produtor utilizou adequadamente ou não o fator de produção (fertilizantes, defensivos, etc.).

Ao invés de usar a produtividade agrícola média dos últimos anos, vários pesquisadores propõem o emprego de modelos probabilísticos que possam ser ajustados aos dados de produtividade de determinada cultura obtidos em um longo período de tempo (ATWOOD et al., 2002, 2003; CARRIQUIRY, BABCOCK e HART, 2008; JUST e WENINGER, 1999; SHERRICK et al., 2004). A partir desse modelo probabilístico pode ser feita a previsão dos pagamentos esperados para o ano subsequente aos dados da série. Esses pesquisadores argumentam que uma modelagem precisa do comportamento dos rendimentos para diversas culturas por distribuições de probabilidade é de fundamental importância para o cálculo das taxas de prêmio do seguro. Assim, devem-se concentrar esforços no sentido de identificar a distribuição que melhor representa os rendimentos da produtividade agrícola para cada tipo de cultura.

O cálculo dos pagamentos esperados e/ou taxas para o seguro agrícola pode levar em conta distribuições de produtividade paramétricas (SHERRICK et al., 2004) e ou não-paramétricas
(OZAKI, GOODWIN e SHIROTA, 2008; TURVEY e ZHAO, 1999). A grande vantagem das densidades paramétricas é que, em geral, seu ajuste não requer uma série de produtividade muito longa. Os métodos não-paramétricos, apesar de serem mais flexíveis para descrever diferentes formas de densidades, não são aplicáveis quando há limitação de dados.

A importância das distribuições de produtividade foi analisada por Zanini et al. (2001), sob o ponto de vista econômico, tendo em vista os valores do seguro. Neste estudo, os autores tiram proveito de um conjunto de dados de alta qualidade, que contém um número relativamente grande de fazendas observadas em um longo período de tempo, na maior região produtora de milho e soja dos Estados Unidos. Os autores mostraram que há grandes diferenças de pagamentos esperados de produtos de seguros agrícolas, dependendo da parametrização escolhida para representar a produtividade.

Just e Weninger (1999) utilizaram extensivas bases de dados de produtividade para defender o uso do modelo normal no cálculo do seguro agrícola. Entretanto, o uso indiscriminado da distribuição normal, para representar a produtividade, vem sendo questionado por diversos pesquisadores.

Atwood et al. (2003) apontaram problemas no trabalho de Just e Weninger (1999) que falharam por não terem considerado que a tendência pode ter introduzido erro do tipo I, ou seja, rejeitando a normalidade quando a distribuição é normal. Atwood et al. (2003) também afirmam que as tendências de estimativas individuais, tendo como base dados de curto prazo, tendem a enviesar a análise também na direção do erro do tipo II, ou seja, falhando ao rejeitar normalidade quando a distribuição é realmente não-normal. Além disso, esses autores destacam os problemas de se trabalhar com um conjunto com poucos dados de produtividade, uma vez que podem ser introduzidos desvios em testes de normalidade.

Sherrick et al. (2004) utilizaram dados de milho e soja de 26 fazendas localizadas na maior região produtora dos Estados Unidos, entre os 
anos de 1972 a 1999, para ajustar as distribuições normal, logística, Weibull, beta e log-normal. Os autores concluíram que as distribuições beta e Weibull forneceram os melhores ajustes, o que sugere a importância de se considerar distribuições que permitam assimetria à esquerda.

Os resultados obtidos por Sherrick et al. (2004) também demonstram que a escolha da distribuição pode ter um impacto significativo nos valores esperados de pagamentos do seguro. Assim, a distribuição de probabilidade da produtividade não deve ser simplesmente aceita como uma premissa não examinada, nem ser escolhida de acordo com a conveniência, sem se avaliar a significância econômica desta suposição.

Ozaki et al. (2008) utilizaram diversos métodos para precificar contratos de seguro agrícola com base na produção por área para dados agregados de produtividade do Instituto Brasileiro de Geografia e Estatística (IBGE), no estado do Paraná. As distribuições paramétricas normal e beta foram ajustadas para as séries de milho, soja e trigo dos municípios de Cascavel, Guarapuava e Tibagi. Os resultados mostraram que as taxas de seguro provenientes da distribuição beta são superiores às taxas da normal para todos os níveis cobertura, considerando as taxas empíricas e as taxas não-paramétricas para milho, soja e trigo. Os resultados sugerem, ainda, alguma assimetria positiva na distribuição beta. Os autores compararam esses resultados com os obtidos utilizando uma abordagem não-paramétrica e concluíram que, para todos os níveis de cobertura, as taxas foram mais elevadas na abordagem não-paramétrica. Isso significa que as companhias de seguros estão subestimando os preços dos contratos de seguro. A taxa de prêmio pura é realmente maior que a taxa de prêmio cobrada. A consequência para a empresa seguradora é a perda financeira devido à menor taxa cobrada. Produtores de alto risco encontram nesta situação atrativa a demanda do contrato de seguro, aumentando a probabilidade de receber a indenização.

Tendo como base o que foi apresentado anteriormente, este trabalho foi realizado com o objetivo de propor a distribuição normal-assimétrica como um modelo alternativo à distribuição normal para a estimação dos pagamentos esperados do seguro agrícola da cultura do milho dentro do cenário agrícola brasileiro. Ademais, considera-se no estudo não o cálculo da taxa de prêmio, mas o cálculo do prêmio em reais por hectare.

\section{Dados de produtividade}

As séries de produtividade analisadas neste trabalho são do período de safras de 1980/1981 a 2006/2007 e foram coletadas em trinta municípios que apresentaram as maiores produtividades de milho no estado do Paraná, no ano de 2007. Optou-se por utilizar apenas as séries dos municípios que apresentavam, no mínimo, 18 anos de observação, no período analisado. Os dados são do rendimento médio da produção da lavoura de milho (em grão) e a unidade é quilogramas por hectare $(\mathrm{kg} / \mathrm{ha})\left(1 \mathrm{ha}=10.000 \mathrm{~m}^{2}\right) \mathrm{e}$ foram coletados pela Secretaria de Agricultura e Abastecimento do Paraná (Seab), nos anos de 1981 a 1989 e pelo Instituto Brasileiro de Geografia e Estatística (IBGE), de 1990 a 2007.

Os trinta municípios selecionados, em ordem decrescente de produtividade de milho (kg/ha), foram Mariópolis, Ibema, Catanduvas, Pato Branco, Castro, Piraí do Sul, Ponta Grossa, Tibagi, Vitorino, Arapoti, Jaguariaíva, Guaraniaçu, Clevelândia, Céu Azul, São João, Sengés, Renascença, Marilândia do Sul, Ipiranga, Campo do Tenente, Nova Prata do Iguaçu, Florestópolis, Mangueirinha, Verê, Palmeira, Campo Bonito, Balsa Nova, Laranjeiras do Sul, Pranchita e Guarapuava.

Espera-se que essas séries de produtividade de milho sejam caracterizadas por apresentarem tendência devido, em parte, ao avanço das tecnologias utilizadas nas lavouras, como técnicas de plantio, maquinário, insumos, etc. Além disso, também se espera que algumas séries possam apresentar dependência temporal e variância não constante. Evidentemente, não é possível ajustar distribuições de probabilidade diretamente aos dados de produtividade que sejam depen- 
dentes e que apresentem tendência temporal. Assim, antes de ajustar os modelos probabilísticos para a produtividade, será necessário utilizar técnicas estatísticas que tornem os dados de produtividade sem tendência, independentes e homocedásticos.

Para verificar a presença de tendência foi utilizado o teste não-paramétrico proposto por Phillips e Perron (1988). O teste considera modelos com um desvio de ajuste e uma tendência temporal para que eles possam ser usados para discriminar entre a raiz unitária não estacionária e acerca de uma tendência determinística estacionária (ou estocasticamente estacionária). $\mathrm{O}$ teste confronta a hipótese nula de que os dados têm raiz unitária contra a hipótese alternativa de que os dados são estocasticamente estacionários.

Uma vez detectada a presença de tendência na série de produtividade, a mesma pode ser retirada por dois métodos, dependendo do tipo da tendência. Quando se tem a presença de tendência determinística, a mesma pode ser retirada com o uso de um modelo polinomial para representar os dados. $\mathrm{O}$ modelo de regressão linear simples é um dos exemplos de modelagem que pode servir para retirar a tendência determinística dos dados e tornar uma série estacionária a partir do uso de seus resíduos (CHARNET et al., 2008). Na presença de tendência estocástica, a mesma pode ser retirada por meio da realização de um processo de passeio aleatório (diferenciação) da série, até obter-se um resíduo sem tendência (EHLERS, 2007).

Uma vez que a série de produtividade está sem tendência, passa-se a verificar a presença de dependência entre as observações, ou seja, o quanto o valor de uma observação influencia nos valores de observações próximas. Em geral, quando se retira a tendência, automaticamente, a série tende a ficar independente. Entretanto, é sempre prudente avaliar a independência dos dados.

Para verificar se uma série apresenta valores independentes, recorre-se a métodos para análise de autocorrelação. Estes métodos verificam o quanto as realizações de uma variável aleatória (observações) estão correlacionadas e, portanto, quantificam a relação de dependência entre as observações. Os métodos mais utilizados para esta finalidade são o teste de Durbin-Watson, descrito em Draper e Smith (1998), e o gráfico da função de autocorrelação (autocorrelograma), descrito em Lages (2004).

Após a execução destes procedimentos, caso não tenha sido detectada autocorrelação serial, prossegue-se com a verificação de possível heterocedasticidade. Caso exista presença de autocorrelação mesmo depois de retirada a tendência (seja pelo modelo de regressão ou pelo processo de passeio aleatório), deve-se desconsiderar o modelo que havia sido ajustado e prosseguir com o ajuste de um modelo autorregressivo.

Um processo autorregressivo (AR) pode ser usado como modelo se for admissível adotar que o valor atual de uma série temporal depende do seu passado imediato mais um erro aleatório. A ordem do modelo AR pode ser definida pela avaliação do gráfico da função de autocorrelação parcial (EHLERS, 2007).

Depois de definida a ordem do modelo AR, é necessário averiguar novamente a presença de autocorrelação serial por meio da elaboração de um novo autocorrelograma para os resíduos obtidos. Deve-se destacar a possibilidade de uso de outros tipos de procedimentos para eliminar a autocorrelação, como, por exemplo, o uso de modelos autorregressivos médias móveis (ARMA) (EHLERS, 2007).

Resumindo, depois da tendência ter sido retirada, havendo presença de dependência entre os dados, a mesma pode ser retirada por meio de um processo autorregressivo até se obter um resíduo independente.

Corrigidas a tendência e a dependência das séries, passa-se a última etapa da preparação dos dados antes do ajuste dos modelos probabilísticos para a produtividade, ou seja, verificar a hipótese de homocedasticidade. Mais uma vez, em geral, quando se retira a tendência, a série pode ficar homocedástica. Entretanto, é sempre prudente verificar a hipótese de homocedasticidade dos dados. 
A fim de se avaliar a presença de heterocedasticidade nas séries de dados, pode-se usar o teste proposto por Breusch e Pagan (1979) que foi originariamente desenvolvido para verificar a presença de distúrbios heterocedásticos em um modelo de regressão linear. A hipótese nula do teste é que a série é homocedástica. Sob a hipótese nula, a estatística do teste segue uma distribuição qui-quadrado com graus de liberdade igual ao número de regressores. Uma forma de obter uma série homocedástica é fazer alguma transformação nos dados descrita em Draper e Smith (1998) e Charnet et al. (2008).

\section{Modelos para a produtividade agrícola}

Com a série de produtividade estacionária, independente e homocedástica é possível ajustar os dados por uma distribuição de probabilidade. Neste artigo serão ajustadas as distribuições normal e normal-assimétrica.

A família de distribuições de probabilidade normal depende de dois parâmetros, a média populacional, denotada por $\mu \mathrm{e}$, a variância também populacional denotada por $\sigma^{2}$. Sua função densidade de probabilidade (ou simplesmente densidade) é dada por Mood et al. (1974).

$$
f(x)=\phi(x)=\frac{1}{\sigma \sqrt{2 \pi}} \exp \left[-(x-\mu)^{2} / 2 \sigma^{2}\right]
$$

sendo $x \in]-\infty ;+\infty[$.

O cálculo de probabilidades da distribuição normal é feito pela integral definida no intervalo da variável aleatória objeto de estudo. O cálculo deve ser realizado utilizando métodos numéricos de integração, pois não existe uma expressão explícita da função densidade de probabilidade acumulada da normal. Não se pode deixar de ressaltar a existência das propriedades de simetria da distribuição normal. Para a modelagem dos dados, na estimação dos parâmetros de média e variância da densidade normal são utilizados estimadores de máxima verossimilhança.

A família de distribuições de probabilidade normal-assimétrica depende do parâmetro de assimetria denotado por $\alpha$. Esta distribuição foi introduzida, pela primeira vez, por O'Hagan e Leonard (1976) para a normal padronizada. No caso da distribuição normal-assimétrica, com parâmetros de locação $[\mu \in \mathbb{R}]$ e escala $[\sigma>0]$, a densidade, reproduzida de Oliveira (2009), é dada por

$$
\mathrm{f}_{\mathrm{Y}}(\mathrm{y})=\frac{2}{\sigma} \phi\left(\frac{\mathrm{y}-\mu}{\sigma}\right) \Phi\left(\alpha \frac{\mathrm{y}-\mu}{\sigma}\right)
$$

em que $y \in \mathbb{R}$. Os símbolos $\phi($.$) e \Phi($. ) são utilizados para denotar, respectivamente, as funções densidade e de distribuição do modelo normal.

Neste estudo, foram utilizados os estimadores de máxima verossimilhança para a estimação dos parâmetros de assimetria, locação e escala da normal-assimétrica. Observa-se, no entanto, que a função de verossimilhança da normal-assimétrica não apresenta forma fechada. Assim, utilizam-se métodos numéricos para a obtenção das estimativas (OLIVEIRA, 2009).

Após o ajuste das distribuições de probabilidade para a produtividade agrícola, testou-se a adequabilidade do ajuste. Para tal, existem diferentes métodos. Neste estudo, foi utilizado o teste de Kolmogorov-Smirnov que investiga a significância da diferença entre a função distribuição empírica observada e a função distribuição de referência (MOOD et al., 1974).

Caso o teste de Kolmogorov-Smirnov indique que as duas distribuições ajustadas podem ser usadas para representar os dados, deve-se definir, entre os dois modelos analisados, aquele que é mais adequado. Para este fim, foi usada a soma de quadrados do erro calculada a partir de cada um dos modelos ajustados para cada uma das séries corrigidas. Utilizou-se como modelo da distribuição de probabilidade aquele que apresentou a menor soma de quadrados do erro.

\section{Cálculo do prêmio de seguro}

Para estimar o pagamento esperado, por unidade de área do seguro agrícola, utilizou-se o Actual Production History (APH) (cálculo da pro- 
dução histórica real) que é apresentado por Ozaki (2005) e Sherrick et al. (2004). O pagamento esperado é usado para fornecer uma estimativa dos valores das indenizações por unidade de área em relação a cada unidade segurada. Em última instância, a teoria econômica do seguro mostra que a indenização esperada pode ser entendida como o prêmio puro de risco. Ou seja, o prêmio sem os carregamentos. Para a utilização do APH, deve-se fazer a união entre as funções densidade de probabilidade das distribuições de produtividade que foram ajustadas e a fórmula que estima o valor do pagamento esperado do seguro.

A seguradora faz um pagamento de indenização a um produtor, caso perceba rendimentos inferiores sob o nível garantido selecionado $(70 \%$, por exemplo) usando a função

$$
\mathrm{Y}_{\mathrm{g}}=\mathrm{h} \times \overline{\mathrm{y}}
$$

em que $Y_{g}$ é a produtividade garantida, h é o nível de cobertura do seguro e y é a produtividade comprovada obtida pela média aritmética simples do histórico das produtividades reais usando, pelo menos, os valores da produtividade de quatro anos anteriores.

Pagamentos de indenizações são acionados quando a produtividade real, $\mathrm{y}$, fica abaixo da produtividade garantida, $\mathrm{Y}_{\mathrm{g}}$, e os produtores recebem como indenização a diferença entre $Y_{g}$ e a produtividade real com um preço garantido que é definido no momento do plantio pela seguradora. Então, a produtividade garantida que ficar abaixo do nível de produtividade esperado tem uma função de pagamento por unidade de área dada por

$$
\mathrm{G}=\max \left\{0, \mathrm{P}_{\mathrm{g}}\left(\mathrm{Y}_{\mathrm{g}}-\mathrm{y}\right)\right\}
$$

sendo $\mathrm{P}_{\mathrm{g}} \mathrm{o}$ preço definido na contratação do seguro.

O pagamento esperado por unidade de área, $\mathrm{E}(\mathrm{G})$, para o $\mathrm{APH}$, pode ser calculado pela expressão

$$
E(G)=\int_{0}^{Y_{g}} \operatorname{Pg}\left(Y_{g}-y\right) f(y) d y
$$

em que f(y) é a densidade do modelo probabilístico ajustado para cada um dos municípios.
Todos os cálculos foram realizados utilizando funções disponíveis e/ou desenvolvidas no R ( $R$ CORE TEAM, 2014). Para obter a densidade, estimativas de máxima verossimilhança e outras funções relativas à distribuição normal-assimétrica foi utilizada a biblioteca fGarch também disponível no R.

\section{Resultados e discussão}

A agricultura é uma atividade suscetível a diversos eventos climáticos como enchentes, granizo, geada, seca, dentre outros. A necessidade do seguro agrícola fica mais evidente diante de problemas climáticos que se apresentam com frequência cada vez maior e trazem grandes prejuízos ao setor. Uma dificuldade enfrentada pelas seguradoras brasileiras é o cálculo das taxas para o seguro agrícola, pois ainda não está disponível uma teoria atuarial consolidada voltada para esta área.

O seguro agrícola mais comercializado no Brasil é o seguro agrícola de custeio que assegura o custeio. Porém, o tipo de seguro analisado refere-se ao seguro de produtividade, que possui muitas semelhanças com o seguro de custeio. A diferença fundamental encontra-se na forma de cálculo do limite máximo de indenização. Ao invés, da multiplicação do custeio por hectare pela área total segurada, utiliza-se a multiplicação do preço do produto, em reais por saca/tonelada, na contratação do seguro, vezes a produtividade segurada, em sacas/toneladas por hectare, vezes a área total segurada, em hectares.

Em geral, as seguradoras brasileiras utilizam a produtividade agrícola média municipal dos últimos quatro anos para estimar os valores das taxas de prêmio e os pagamentos esperados do seguro. Esta prática é criticada por diversos pesquisadores tais como Atwood et al. (2002, 2003), Carriquiry et al. (2008), Just e Weninger (1999), Lawas (2005), Ozaki (2005) e Sherrick et al. (2004) que argumentam que uma modelagem precisa do comportamento dos rendimentos para diversas culturas, por distribuições de probabilidade, é 
de fundamental importância para a precificação adequada do contrato de seguro.

O não uso de modelos que descrevem a produtividade agrícola brasileira para o cálculo dos pagamentos esperados do seguro pode ser creditado não somente à limitada quantidade de estudos sobre modelos que sejam adequados à produtividade brasileira, mas também à falta de dados apropriados para esta finalidade.

Alguns autores, como Sherrick et al. (2004), sugerem que os dados para o estudo da produtividade devem ter algumas características, tais como (a) alta qualidade; (b) série suficientemente longa (ter, no mínimo, vinte observações no período amostral usado no estudo, para que as séries possam refletir, precisamente, a estrutura de risco dos produtores); (c) grande número de fazendas e (d) fazendas com características parecidas em relação à irrigação, latitude, longitude e etc.

Embora sejam feitas as recomendações anteriores, na prática, no Brasil não se tem informações oficiais com uma caracterização sobre as fazendas. Existem apenas séries suficientemente longas de dados de produtividade de algumas culturas em nível de município. Na Tabela 1 são mostradas algumas estatísticas descritivas para os

Tabela 1. Estatísticas descritivas das séries originais de produtividade de milho (quilogramas/hectare) para trinta municípios do Paraná, 1981-2007

\begin{tabular}{|c|c|c|c|c|c|}
\hline Município & Obs. (n) & Média & Desvio padrão & Assimetria & Curtose \\
\hline Mariópolis & 27 & 4357,565 & 2273,400 & 0,6686472 & 2,067108 \\
\hline Ibema & 18 & 5268,722 & 1626,502 & 0,2700403 & 2,412835 \\
\hline Catanduvas & 27 & 4770,456 & 2123,013 & 0,3740965 & 1,869979 \\
\hline Pato Branco & 27 & 4107,510 & 2148,411 & 0,6745335 & 2,026116 \\
\hline Castro & 27 & 5978,389 & 1448,195 & 0,1140864 & 1,971467 \\
\hline Piraí do Sul & 27 & 4063,348 & 2055,995 & 0,5932287 & 2,372392 \\
\hline Ponta Grossa & 27 & 5358,468 & 1679,890 & 0,04140333 & 2,086141 \\
\hline Tibagi & 27 & 4873,462 & 1996,684 & 0,0689475 & 1,706156 \\
\hline Vitorino & 27 & 4372,337 & 2500,260 & 0,69881 & 1,98233 \\
\hline Arapoti & 27 & 4375,483 & 1839,167 & 0,8017741 & 2,881228 \\
\hline Jaguariaíva & 27 & 2901,867 & 1999,567 & 1,623389 & 4,381107 \\
\hline Guaraniaçu & 27 & 3409,566 & 1692,622 & 1,037457 & 3,328625 \\
\hline Clevelândia & 27 & 4085,142 & 2005,933 & 0,4576608 & 1,849243 \\
\hline Céu Azul & 27 & 4559,774 & 1763,926 & 0,4769858 & 2,203402 \\
\hline São João & 27 & 3693,539 & 1758,127 & 0,8298935 & 2,699335 \\
\hline Sengés & 27 & 3082,747 & 1785,116 & 0,9972375 & 3,195999 \\
\hline Renascença & 27 & 4127,515 & 1846,894 & 0,4175055 & 1,911589 \\
\hline Marilândia do Sul & 27 & 4925,508 & 2043,735 & 0,03713805 & 1,601507 \\
\hline Ipiranga & 27 & 3762,981 & 1666,904 & 0,5703219 & 2,409658 \\
\hline Campo do Tenente & 27 & 3156,159 & 1920,101 & 1,141168 & 2,935735 \\
\hline Nova Prata Iguaçu & 25 & 3531,697 & 1279,085 & 1,54191 & 5,456018 \\
\hline Florestópolis & 27 & 3486,437 & 1094,655 & 1,880815 & 8,1579 \\
\hline Mangueirinha & 27 & 3912,896 & 1780,167 & 0,4770354 & 1,871744 \\
\hline Verê & 27 & 3244,712 & 1657,374 & 1,3427240 & 3,438708 \\
\hline Palmeira & 27 & 4008,290 & 1632,307 & 0,5411703 & 2,046826 \\
\hline Campo Bonito & 19 & 4758,316 & 1779,731 & 0,2440458 & 1,488304 \\
\hline Balsa Nova & 27 & 3021,058 & 1827,154 & 1,1680970 & 3,148854 \\
\hline Laranjeiras do Sul & 27 & 2988,614 & 1606,081 & 1,3728760 & 4,003556 \\
\hline Pranchita & 25 & 3300,814 & 1288,937 & 1,0385950 & 4,414578 \\
\hline Guarapuava & 27 & 4126,372 & 1976,647 & 0,1800924 & 1,598059 \\
\hline Média & 26,3 & 4053,658 & 1803,219 & 0,7227723 & 2,783883 \\
\hline Mínimo & 18 & 2901,867 & 1094,655 & 0,0371380 & 1,488304 \\
\hline Máximo & 27 & 5978,389 & 2500,260 & 1,8808150 & 8,157900 \\
\hline
\end{tabular}

Fonte: Resultados da pesquisa. 
dados de produtividade de milho dos municípios analisados a partir das séries brutas (sem a retirada da tendência).

A Tabela 1 mostra que as produtividades médias de milho entre os municípios foi 4053,6 kg/ha, com uma produtividade mínima de 2901,8 $\mathrm{kg} / \mathrm{ha}$ e máxima de 5978,3 kg/ha. A variabilidade da produtividade média de milho difere entre os municípios amostrados com desvios padrão que vão de 1094,6 a 2500,2kg/ha. Todas as séries de produtividade de milho do Paraná exibiram assimetria positiva, ou seja, à direita. Os coeficientes de assimetria variaram de 0,03 a 1,88 com uma média de 0,72 . A maioria das séries $(70 \%)$ foram classificadas como platicúrticas.

Na Figura 1, a seguir, são mostrados os gráficos das séries originais de produtividade de milho (kg/ha) para cada um dos municípios considerados.

Deve-se ressaltar que a teoria atuarial de segurabilidade é baseada em alguns pressupostos tais como independência entre os eventos e homocedasticidade. Entretanto, conforme explicitado na revisão de literatura, existe um risco sistêmico associado ao seguro agrícola e a pressuposição de independência entre as unidades seguradas é violada, fazendo com que o risco agregado seja maior que o risco individual (OZAKI, 2005, 2007). Este fato não somente dificulta a quantificação dos riscos associados à atividade, mas também impede o uso de modelos probabilísticos diretamente nas séries brutas, para o cálculo do pagamento esperado do seguro.

A Figura 1 mostra a presença de tendência nas séries de produtividade de todos os municípios analisados. Essa tendência se deve em parte, ao fato do avanço das tecnologias utilizadas nas lavouras de milho, como técnicas de plantio, maquinário, insumos, etc. A presença de tendência nas séries de produtividade analisadas contrasta com as séries de produtividade de milho, praticamente estacionárias, observadas na maior região produtora dos Estados Unidos (SHERRICK et al., 2004).

Apesar da inspeção visual dos gráficos da Figura 1 indicar a presença de algum tipo de tendência (estocasticamente estacionária/determinística ou estocástica), também se adotou o teste de Phillips-Perron para o mesmo propósito. Segundo Sherick et al. (2004) este teste é uma das abordagens mais comuns para testar a existência de tendência estocástica. Enders (1995) e Sherrick et al. (2004) argumentam em favor do teste para avaliar a possibilidade de tendência estocasticamente estacionária, pois permite um conjunto mais fraco de hipóteses sobre o processo de erro e apresenta um maior poder para rejeitar uma hipótese nula falsa de uma raiz unitária. O teste de Phillips-Perron rejeitou a hipótese de estacionaridade $(p>0,05)$ para dezesseis das trinta séries analisadas. Assim, foram utilizadas as técnicas de regressão linear simples, passeio aleatório (diferenciação da série) e processos auto-regressivos de ordem um para contornar o problema de tendência das séries de produtividade de acordo com o tipo de tendência encontrada.

Deve-se observar que o número de observações da série sem tendência pode diferir do número de observações da série original de acordo com o método utilizado na retirada da tendência. Por exemplo, a cada vez que um modelo de passeio aleatório simples é utilizado para se obter uma série sem tendência, a série original perde uma observação.

Após empregar as técnicas de regressão linear simples, diferenciação da série e processos auto-regressivos, dependendo do tipo de tendência observada na série de produtividade, novamente, foi realizada a análise de tendência. $O$ teste de Phillips-Perron aceitou a hipótese de série estocasticamente estacionária $(p<0,05)$ para todas as séries analisadas.

Retirada a tendência dos dados, verificou-se a hipótese de independência entre as observações através do uso do teste de Durbin-Watson e do gráfico da função de autocorrelação. $O$ teste de Durbin-Watson mostrou que, com exceção dos municípios de Castro, Mangueirinha e Guarapuava, os demais apresentaram séries com dados independentes ( $p>0,05)$. Os autocorrelogramas mostraram que os municípios de Piraí do Sul, Tibagi, Jaguariaíva, Sengés, Campo 


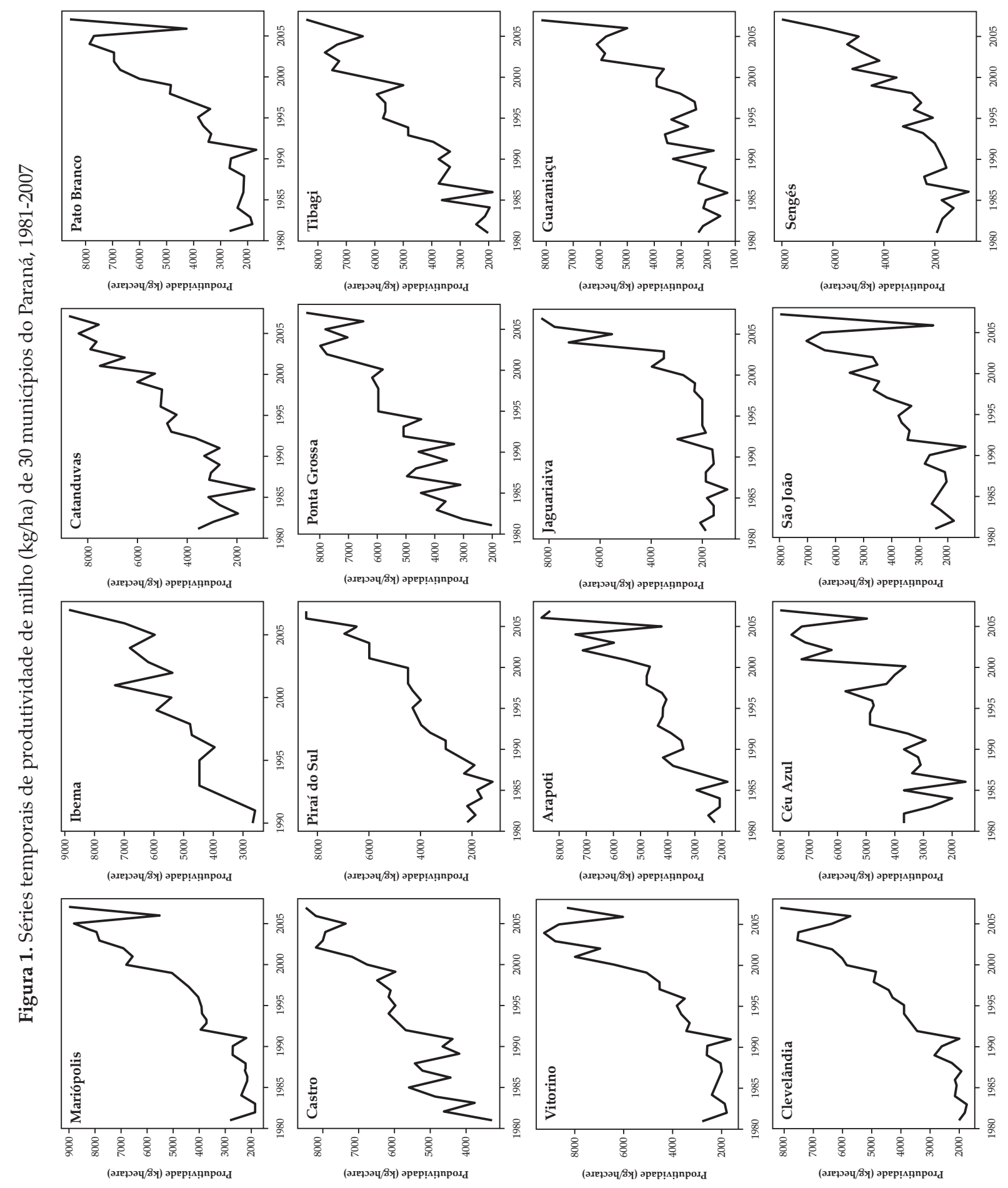



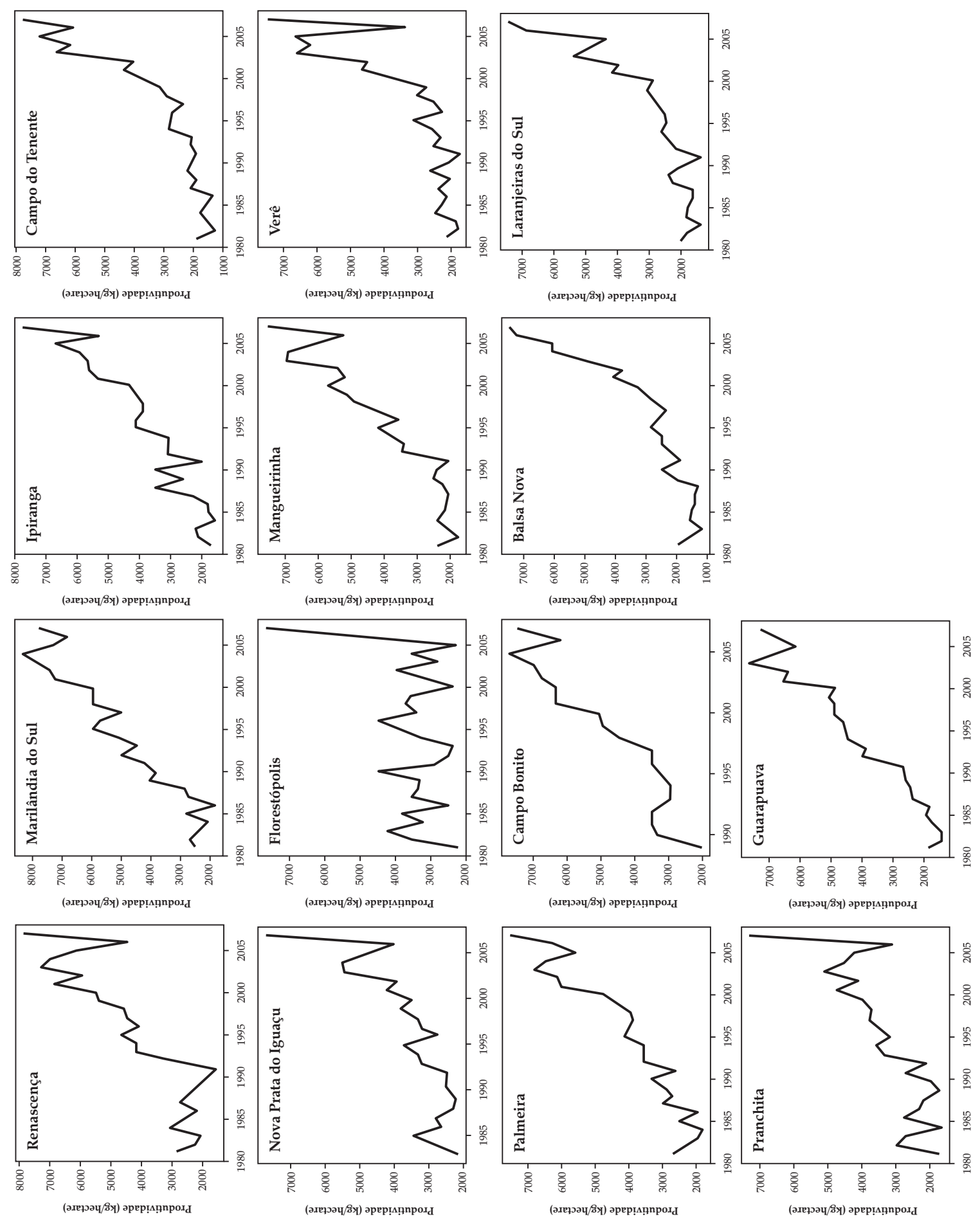
do Tenente e Verê apresentaram problemas de dependência. Neste caso, para estas cidades foi usado um modelo AR(1).

Corrigidas a tendência e a dependência das séries, passou-se a última etapa da preparação dos dados, antes do ajuste das densidades, ou seja, verificar a hipótese de homocedasticidade. $\mathrm{O}$ teste de Breusch-Pagan mostrou que as séries de produtividade eram homocedásticas ( $\mathrm{p}>$ 0,05), com exceção dos municípios de Arapoti e São João, onde foi detectada apenas a presença de uma heterocedasticidade leve e, portanto, fez-se a opção de não realizar transformações para corrigir a variância dessas cidades.

Com a série corrigida é possível modelar os dados por uma distribuição de probabilidade. A Figura 2, a seguir, mostra as densidades normal e normal-assimétrica ajustadas para as séries corrigidas de produtividade de milho ( $\mathrm{kg} / \mathrm{ha}$ ) dos municípios do Paraná.

Observa-se na Figura 2 que para os municípios de Céu Azul, Jaguariaíva, Laranjeiras do Sul, Nova Prata do Iguaçu e Ponta Grossa não houve convergência dos estimadores de máxima verossimilhança da normal-assimétrica e, portanto, impossibilitou o seu ajuste.

Após o ajuste das densidades normal e normal-assimétrica para as distribuições de produtividade agrícola, testou-se a adequabilidade dos ajustes utilizando o teste de KolmogorovSmirnov (ks). A densidade normal não apresentou ajuste estatisticamente significante, ao nível de $10 \%$, apenas para a distribuição de produtividade do município de Jaguariaíva, por este motivo, não foi apresentado na Figura 2 o gráfico relativo ao referido município. A densidade normal-assimétrica apresentou ajuste estatisticamente significante, ao nível de $10 \%$, para todos os municípios em que a densidade foi ajustada.

A Tabela 2 mostra a soma dos quadrados dos erros de cada uma das séries corrigidas para as distribuições normal e normal-assimétrica ajustadas.

A Tabela 2 mostra que a densidade normal-assimétrica apresentou as menores somas de quadrados dos erros para todas as distribuições de produtividade dos municípios em que foi possível ajustar a densidade. Assim, pode-se concluir que a normal-assimétrica foi a distribuição com melhor ajuste para todas as séries em que foi possível o ajuste das duas distribuições analisadas.

Na última etapa do processo, o APH é usado para fornecer estimativas dos valores esperados das indenizações por unidade de área em relação a cada unidade segurada. Neste caso é feita a união entre a função densidade de probabilidade da distribuição de produtividade que foi ajustada e a fórmula que estima o valor do pagamento esperado do seguro (equação 5).

A Tabela 3 mostra os valores estimados (em $\mathrm{R} \$$ /ha) para o pagamento esperado do seguro agrícola para cada uma das cidades selecionadas do Paraná com base no APH. Para obter os valores estimados foi usado o indicador Cepea/Esalq - Milho (31-01-2011) com um preço do milho de $\mathrm{R} \$ 32,11$ por saca de 60 quilogramas para ilustrar os cálculos e simular o preço do milho previsto em contrato.

Os valores estimados apresentados na Tabela 3 mostram estimativas para a indenização esperada por unidade de área (ha). Os valores estimados mostram, em moeda corrente ( $R \$)$, a diferença entre a produtividade real e a produtividade esperada, prevista em contrato, que a seguradora deverá "completar" em dinheiro caso ocorra sinistro no município. Por exemplo, no município de Catanduvas a previsão é que a seguradora pagará uma indenização no valor de $\mathrm{R} \$ 14,45$, por hectare, de acordo com a distribuição normal e de $\mathrm{R} \$ 16,61$ de acordo com a normal-assimétrica.

A Tabela 3 mostra que utilizando o APH, os pagamentos esperados com o uso da distribuição normal-assimétrica foram maiores que os estimados pela distribuição normal em 9 dos 25 municípios com um nível de $70 \%$ de cobertura. Este fato sugere alguma assimetria positiva na distribuição normal-assimétrica. Estes resultados corroboram os resultados obtidos por Ozaki et al. (2008) que apontaram, para os municípios de Cascavel, Guarapuava e Tibagi, alguma assimetria positiva com o uso da distribuição beta. 
Tabela 2. Valores das somas dos quadrados dos erros para as distribuições normal e normal-assimétrica ajustadas para as séries corrigidas dos municípios do Paraná

\begin{tabular}{|c|c|c|}
\hline Município & Normal & $\begin{array}{c}\text { Normal- } \\
\text {-assimétrica }\end{array}$ \\
\hline Mariópolis & 21864284 & 21864278 \\
\hline Ibema & 7446391 & 7446387 \\
\hline Catanduvas & 17111482 & 17111476 \\
\hline Pato Branco & 25778779 & 25778775 \\
\hline Castro & 7999119 & 7999114 \\
\hline Piraí do Sul & 10798113 & 10798108 \\
\hline Ponta Grossa & 11952740 & -- \\
\hline Tibagi & 8728477 & 8728473 \\
\hline Vitorino & 29674761 & 29674757 \\
\hline Arapoti & 19266160 & 19266155 \\
\hline Jaguariaíva & ks & -- \\
\hline Guaraniaçu & 29613430 & 29613424 \\
\hline Clevelândia & 13860732 & 13860729 \\
\hline Céu Azul & 27501327 & -- \\
\hline São João & 27248751 & 27248748 \\
\hline Sengés & 18469095 & 18469090 \\
\hline Renascença & 28301750 & 28301744 \\
\hline Marilândia do S. & 12325812 & 12325809 \\
\hline Ipiranga & 9847609 & 9847602 \\
\hline Campo do Tenente & 12814034 & 12814028 \\
\hline Nova Prata do Iguaçu & 22291792 & -- \\
\hline Florestópolis & 34581821 & 34581814 \\
\hline Mangueirinha & 10595520 & 10595515 \\
\hline Verê & 23673437 & 23673434 \\
\hline Palmeira & 9493398 & 9493392 \\
\hline Campo Bonito & 10290144 & 10290142 \\
\hline Balsa Nova & 7033049 & 7033044 \\
\hline Laranjeiras do S. & 13740905 & -- \\
\hline Pranchita & 14685681 & 14685677 \\
\hline Guarapuava & 5956707 & 5956702 \\
\hline
\end{tabular}

(--) Não houve convergência do estimador de máxima verossimilhança da normal-assimétrica.

(ks) Teste de Kolmogorov-Smirnov não apresentou ajuste estatisticamente significante, ao nível de $10 \%$.

Fonte: Resultados da pesquisa.

As distribuições normal e normal-assimétrica foram escolhidas para este estudo por apresentarem a possibilidade de ajuste de uma série que contenha valores de observações positivos e negativos. Conforme apresentado na introdução, existem outras distribuições como a log-normal, beta, etc. que poderiam ser testadas. Entretanto, as séries de produtividade utilizadas neste trabalho apresentaram tendência, o que exigiu métodos de correção das mesmas. Sabe-se que as séries corrigi-
Tabela 3. Pagamento esperado (em Reais por hectare) por unidade de área da cultura de milho para cidades do Paraná, cobertura de 70\%, 1981-2007

\begin{tabular}{|c|c|c|}
\hline Município & Normal & $\begin{array}{c}\text { Normal- } \\
\text {-assimétrica }\end{array}$ \\
\hline Mariópolis & 10,70493 & 12,07344 \\
\hline Ibema & 0,1985859 & 0,1978225 \\
\hline Catanduvas & 14,45295 & 16,61428 \\
\hline Pato Branco & 2,809048 & 2,917147 \\
\hline Castro & 0,7567108 & 0,7932405 \\
\hline Piraí do Sul & 54,65214 & 40,44970 \\
\hline Ponta Grossa & 1,410876 & -- \\
\hline Tibagi & 5,540277 & 5,27399 \\
\hline Vitorino & 0,5070298 & 0,4216994 \\
\hline Arapoti & 11,41671 & 12,72312 \\
\hline Jaguariaíva & ks & -- \\
\hline Guaraniaçu & 17,49157 & 15,47649 \\
\hline Clevelândia & 1,802261 & 1,167653 \\
\hline Céu Azul & 6,783582 & -- \\
\hline São João & 3,606921 & 3,934251 \\
\hline Sengés & 68,44152 & 63,05922 \\
\hline Renascença & 1,028588 & 0,7941647 \\
\hline Marilândia do S. & 0,01902890 & 0,01532165 \\
\hline Ipiranga & 15,57037 & 18,882 \\
\hline Campo do Tenente & 23,80958 & 14,3778 \\
\hline Nova Prata do Iguaçu & 18,31154 & -- \\
\hline Florestópolis & 66,65323 & 77,0081 \\
\hline Mangueirinha & 1,825512 & 1,947399 \\
\hline Verê & 0,01780649 & 0,01551144 \\
\hline Palmeira & 2,646129 & 1,982869 \\
\hline Campo Bonito & 2,487175 & 1,893065 \\
\hline Balsa Nova & 34,2689 & 27,84263 \\
\hline Laranjeiras do S. & 18,24871 & -- \\
\hline Pranchita & 0,01203237 & 0,01201180 \\
\hline Guarapuava & 4,559823 & 4,075864 \\
\hline
\end{tabular}

(--) Não houve convergência do estimador de máxima verossimilhança da normal-assimétrica.

(ks) Teste de Kolmogorov-Smirnov não apresentou ajuste estatisticamente significante, ao nível de $10 \%$.

Fonte: Resultados da pesquisa.

das podem apresentar valores negativos, fato que impossibilita o uso de algumas distribuições paramétricas, como é o caso da densidade log-normal.

Deve-se destacar que o modelo normal não pode deixar de ser examinado, pois a distribuição normal é utilizada frequentemente em vários artigos da área, tais como Atwood, et al. (2002 e 2003), Just e Weninger (1999), Kapiamba (2005), Ramírez et al. (2001), Ozaki et al. (2008), Sherrick et al. (2004) e Zanini et al. (2001), entre outros. 

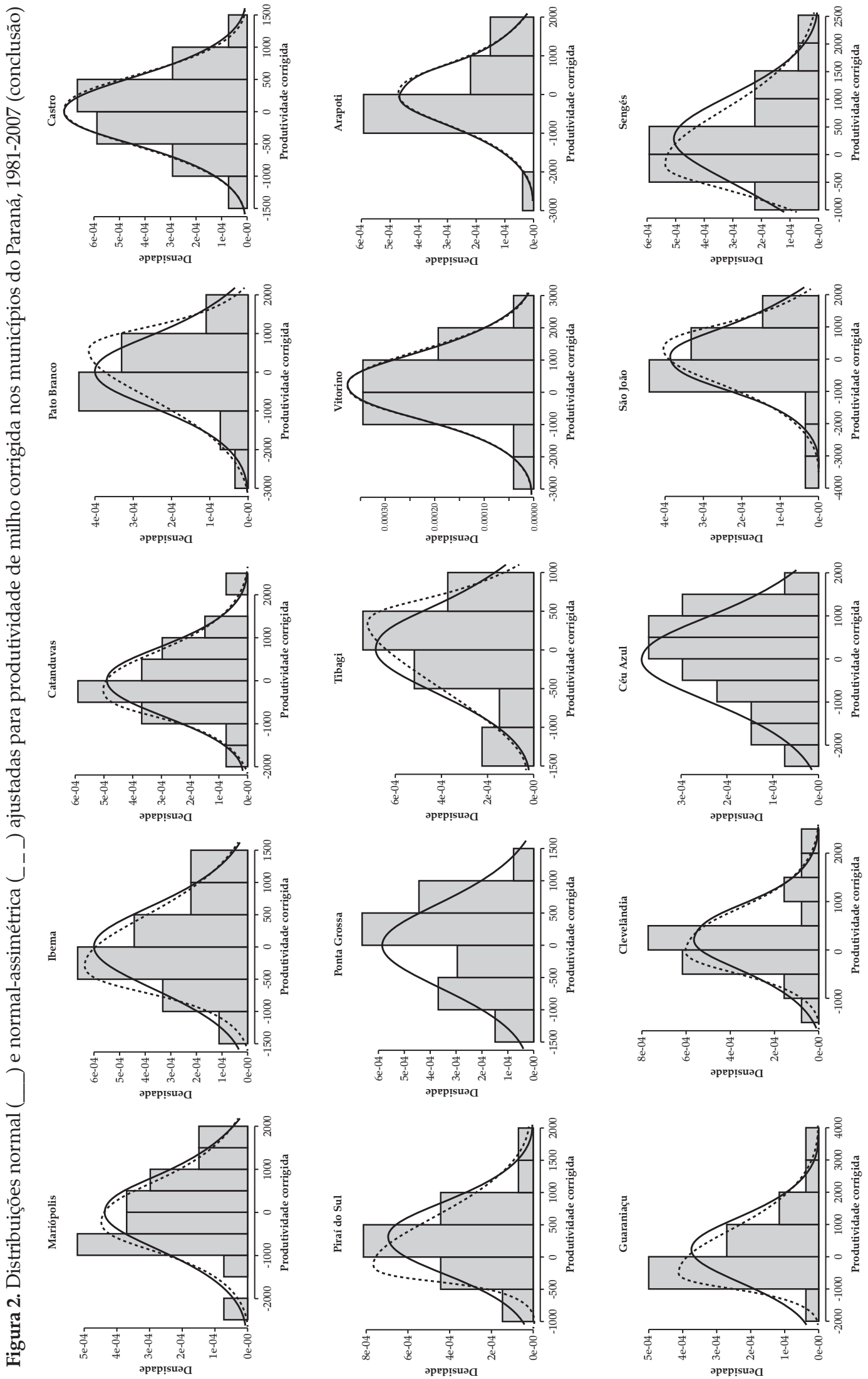

RESR, Piracicaba-SP, Vol. 52, № 04, p. 725-742, Out/Dez 2014 - Impressa em Janeiro de 2015 

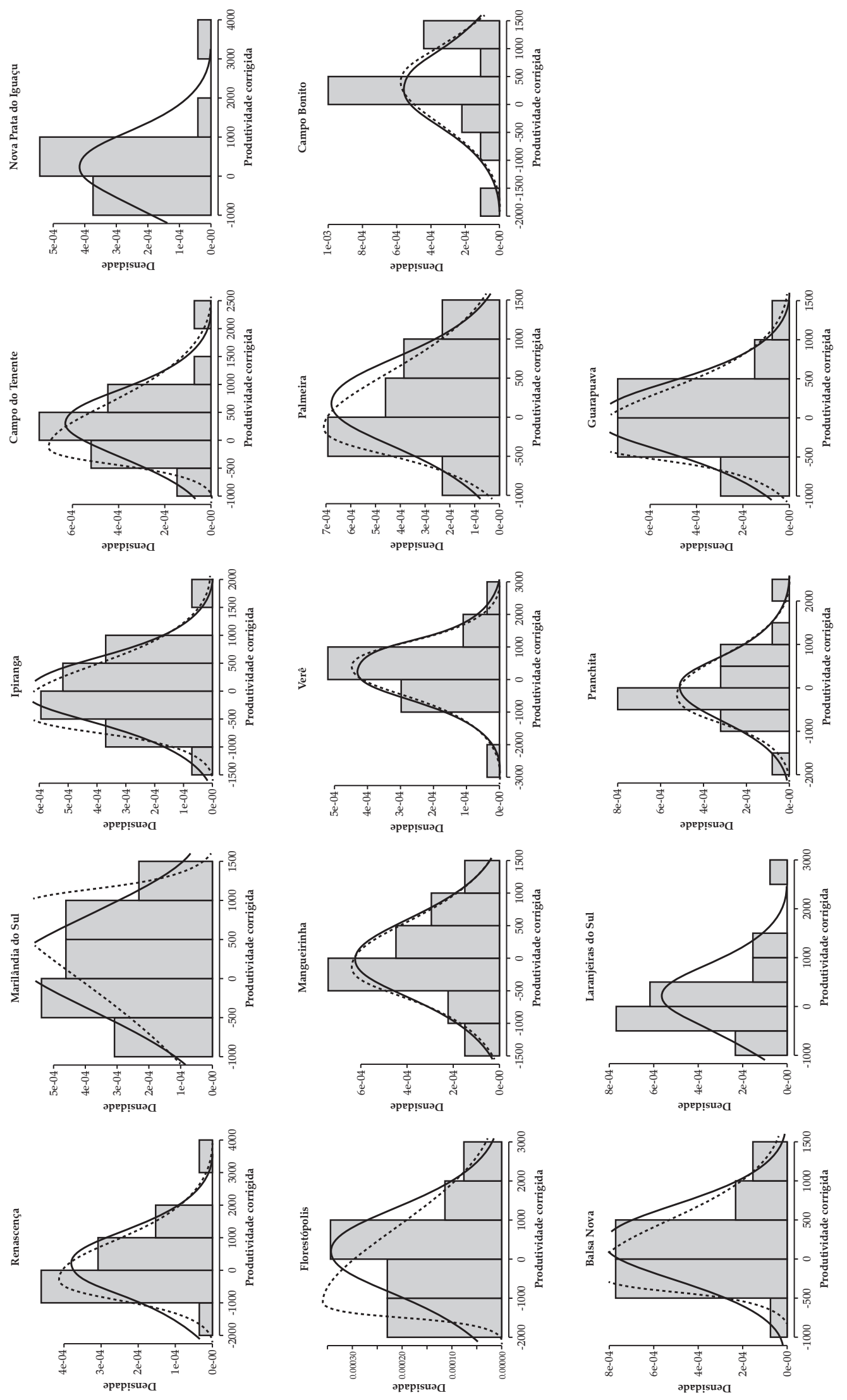

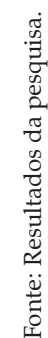

RESR, Piracicaba-SP, Vol. 52, № 04, p. 725-742, Out/Dez 2014 - Impressa em Janeiro de 2015 
Uma vantagem da distribuição normal-assimétrica em relação à normal é a possibilidade de assimetria. Uma desvantagem da distribuição normal-assimétrica em relação à normal é possibilidade de não ocorrer convergência no algoritmo que estima os parâmetros do modelo. Ressalta-se que ambos os modelos apresentam uma limitação que é a impossibilidade de bimodalidade ou multimodalidade da distribuição de produtividade.

Observa-se que a distribuição normal-assimétrica apresenta alguma assimetria positiva em relação à normal e, portanto, sugere que a normal estaria subestimando o pagamento esperado em alguns municípios. O prêmio leva em conta o valor do pagamento esperado, como consequência, pode-se subestimá-lo e cobrar menos do que o necessário para compensar as perdas e ainda, em caso de sinistro, a seguradora deverá fazer um pagamento maior do que o previsto.

Proprietários de fazendas com maior probabilidade de ocorrência de sinistro encontram no cenário descrito acima um atrativo para fazer o seguro de produtividade de suas lavouras. Estes resultados corroboram o estudo de Ozaki e Silva (2009) que usaram um método alternativo baseado em modelos hierárquicos bayesianos sob a suposição da distribuição normal-assimétrica para precificar contratos de seguro agrícola para municípios do Paraná e concluíram que os métodos utilizados para este fim no mercado de seguros subestimam os prêmios, podendo trazer prejuízos às seguradoras.

Deve-se observar que neste estudo foram utilizados dados, em nível de município, logo o seguro teria os mesmos prêmios e valores estimados de indenizações para cada unidade de área, de cada fazenda, dentro do mesmo município. Este fato não permite a avaliação de diferenças entre as fazendas.

Outro fato que deve ser destacado é o modo de pagamento das indenizações aos segurados. Após a constatação de sinistro no seguro agrícola, as seguradoras pagam as indenizações "completando" em dinheiro o percentual de produtividade que ficou abaixo do nível contratado a um preço estabelecido previamente no contrato.
Finalmente, pode-se destacar que este estudo propôs a densidade normal-assimétrica como uma alternativa para modelar dados de produtividade agrícola no Brasil que, até o momento, não foi considerada em trabalhos teóricos para o cálculo dos pagamentos esperados do seguro. Embora não tenha sido possível comparar os resultados obtidos com dados reais de pagamentos esperados de seguradoras, de maneira geral, pode-se concluir que a distribuição normal-assimétrica forneceu um melhor ajuste que a distribuição normal para a produtividade de milho nos municípios analisados. Espera-se que os resultados possam contribuir para o progresso do seguro agrícola brasileiro e que sejam úteis para outros pesquisadores da área.

\section{Conclusões}

Neste trabalho apresentou-se, pela primeira vez, a distribuição normal-assimétrica como um modelo alternativo para a estimativa do pagamento esperado de seguro agrícola no Brasil. Os resultados mostraram que a distribuição normal-assimétrica é um modelo competitivo para explicar as distribuições de produtividade agrícola de milho e, portanto, pode ser utilizada para o cálculo dos pagamentos esperados no seguro agrícola de milho no Brasil. Assim, a distribuição normal-assimétrica pode substituir, para esse fim, não somente a distribuição normal, mas também o método da produtividade média dos anos anteriores.

\section{Referências bibliográficas}

ATWOOD, J., SHAIK, S. E WATTS, M. Can normality of yields be assumed for crop insurance? Canadian Journal of Agricultural Economics, v. 50, p. 171-184, 2002.

., SHAIK, S. e WATTS, M. Are Crop Yields Normally Distributed? A Reexamination. American Journal of Agricultural Economics, v. 85, p. 888-901, nov. 2003.

BREUSCH, T. S. e PAGAN, A. R. A simple test for heteroscedasticity and random coefficient variation. Econometrica, v. 47, n. 5, 1979. 
CARRIQUIRY, M. A., BABCOCK, B. A. e HART, C. E. Using a farmer's beta for improved estimation of expected yields. Journal of Agricultural and Resource Economics, v. 33, p. 52-68, 2008.

CHARNET, R. et al. Análise de modelos de regressão linear com aplicações. 2. ed. Campinas, SP: Ed. Unicamp, 2008. 368 p.

COBLE, K.H. et al. An Expected Indemnity Approach to the Measurement of Moral Hazard in Crop Insurance. American Journal of Agricultural Economics, v. 79, p. 21626, fev. 1997.

DRAPER, N. R. e SMITH, H. Applied Regression Analisys. 3. ed. New York: Ed. John Willey e Sons, 1998. 704 p.

EHLERS, R. S. Análise de séries temporais. Laboratório de Estatística e Geoinformação, Universidade Federal do Paraná, 4. ed., 2007.

ENDERS, W. Applied econometric time series. 1. ed. New York: John Wiley \& Sons, 480 p., 1995.

INDICADOR CEPEA/ESALQ - Milho (Fonte: Cepea). Preço Milho em 31-01-2011: R \$32,11 por saca de 60 $\mathrm{kg}$ com variação de $+0,03 \%$, fechamento em 31-012011. BRASIL (BM\&F). Disponível em: <http://www. noticiasagricolas.com.br/cotacoes.php?id $=20>$. Acesso em: 1 fev. 2010.

JUST, R. E., CALVIN, L. e QUIGGIN, J. Adverse selection in crop insurance: actuarial and asymmetric information incentives. American Journal of Agricultural Economics, v. 81, p. 834-849, 1999.

. e WENINGER, Q. Are Crop Yields Normally Distributed?. American Journal of Agricultural Economics, v. 81, p. 278-304, 1999.

KAPIAMBA, L. F. Modeling Heteroskedasticity of Crop Yield Distributions: Implications for Normality. Selected Paper Prepared for Presentation at the American Agricultural Economics Association Annual Meeting, Providence, Rhode Island, July 24-27, 2005.

LAGES, W. F. Descrição de sinais aleatórios. Universidade Federal do Rio Grande do Sul, Programa de Pós-Graduação em Engenharia Elétrica, Disciplina ELE00071-Tópicos Especiais em Automação e Controle II, 2004. Disponível em: <www.ece.ufrgs.br/ fetter/ ele00071/dec/signals.pdf > . Acesso em: 24 jan. 2011.

LAWAS, C. P. B.S. Crop Insurance Premium Rate Impacts of Flexible Parametric Yield Distributions: an Evaluation of the Johnson Family of Distributions. 2005. 81 p.. Tese. (Mestrado em Ciências) Texas Tech University, 2005.

MOOD, A. M., GRAYBILL, F. A. e BOES, D. C. Introduction to the Theory of Statistics. Ed. McGraw-Hill, 577 p. 1974.
MORETTIN, P. A. e TOLOI, C. M. C. Análise de Séries Temporais. 2. ed. São Paulo: Egard Blucher, 538 p., 2006.

O'HAGAN, A. e LEONARD, T. Bayes estimation subject to uncertainty about parameter constraints. Biometrika, v. 63, n. 1, p. 201-203, 1976.

OLIVEIRA, M. S. de. Comparações Múltiplas Bayesianas com Erro Normal assimétrico. 2009.154 p. Tese. (Doutorado em Estatística e Experimentação Agropecuária). Universidade Federal de Lavras, Lavras, 2009.

OZAKI, V. A. Métodos atuariais aplicados à determinação da taxa de prêmio de contratos de seguro agrícola: um estudo de caso. 2005. 324 p. Tese (Doutorado em Economia Aplicada). Escola Superior de Agricultura "Luiz de Queiroz", Piracicaba, SP, 2005.

O governo federal e o mercado de seguro agrícola: aprendendo com o passado e construindo o futuro. In: XLIV Congresso da Sociedade Brasileira de Economia e Sociologia Rural (SOBER), 2006, Fortaleza. XLIV Congresso da Sociedade Brasileira de Economia e Sociologia Rural (SOBER), 2006.

. O papel do seguro na gestão do risco agrícola e os empecilhos para o seu desenvolvimento. Revista Brasileira de Risco e Seguro (online), v. 2, p. 75-92, 2007.

., GOODWIN, B. K. e SHIROTA, R. Parametric and non-parametric statistical modeling of crop yield: implications for pricing crop insurance contracts. Applied Economics, v. 40, p. 1151-1164, 2008.

. e SILVA, R. S. Bayesian ratemaking procedure of crop insurance contracts with skewed distribution. Journal of Applied Statistics. v. 36, p. 443-452, 2009.

PHILLIPS, P. C. B. e PERRON, P. Testing for a unit root in time series regression. Biometrika, v. 75, n. 2, p. 335346, 1988.

R CORE TEAM. $R$ : a language and environment for statistical computing. Vienna, Austria: R Foundation for Statistical Computing, 2014. Disponível em: <http:// www.R-project.org >.

RAMÍREZ, O. A., MISRA, S. e FIELD, J. Are crop yieds normally distributed? Annual meeting of the American Agricultural Economics Association, Chicago, Illinois, August 05-08, 2001.

SHERRICK, B. J., ZANINI, F. C., SCHNITKEY, G. D. e IRWIN, S. H. Crop Insurance Valuation under Alternative Yield Distributions. American Journal of Agricultural Economics, v. 86, n. 2, p. 406-419, 2004. 
742 - A Distribuição Normal-assimétrica como Modelo para Produtividade de Milho Aplicada ao Seguro Agrícola

TURVEY, C. G. e ZHAO, J. Parametric and nonparametric crop yield distributions and their effects on all-risk crop insurance premiums. Working Paper WP99/05. Department of Agricultural Economics and Businees. University of Guelph, 1999.
ZANINI, F. C. et al. Crop insurance valuation under alternative yield distributions. Proceedings of the NCR134 Conference on Applied Commodity Price Analysis, Forecasting, and Market Risk Management. St. Louis, MO. 2001. [http://www.farmdoc.uiuc.edu/nccc134]. 